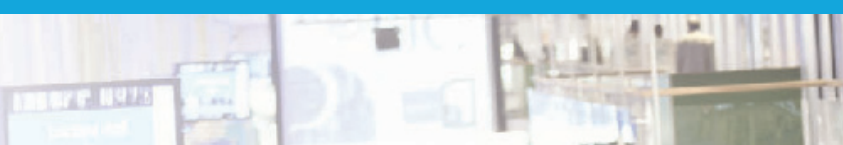

\title{
European Multidisciplinary Cancer Congress
}

Der diesjährige Kongress der European Cancer Organisation (ECCO) stand unter dem Thema Multidisziplinarität und wurde zu der European Society for Medical Oncology (ESMO) und derEuropean Society for Therapeutic Radiology and Oncology (ESTRO) veranstalte Das Kongressprogramm deckte das ganze Themenspektrum, d.h. die Bereiche Grundlagen- und translationale Forschung, Prävention, Behandlung, Pflege und supportive Behandlung, zu den verschiedenen Tumorerkrankungen ab. Schwerpunkte dabei waren insbesondere die, Bedeutung der Multidisziplinarität in z.B. Forschung und Behandlungsalltag, aber auch die Berücksichtigung der Patientenperspektive bei allen Bemühungen und die personalisierte Medizin.
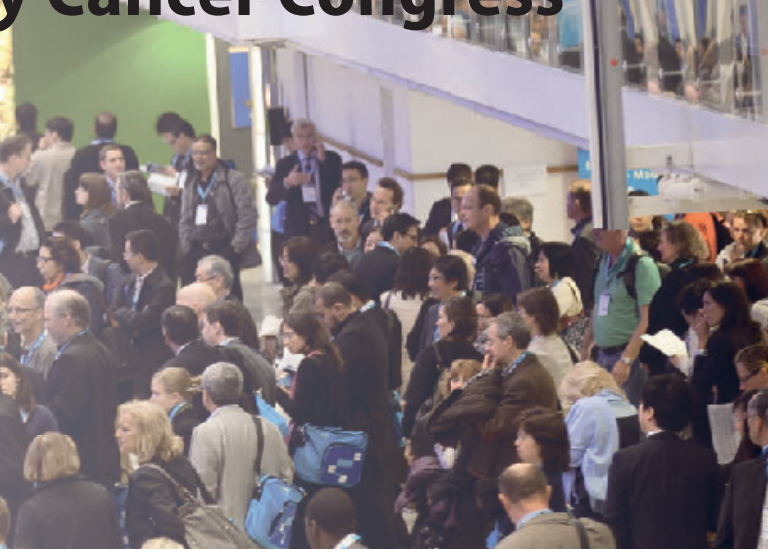

\section{Kolorektale Lebermetastasen}

\section{Längeres Überleben durch R0-Resektion nach Cetuximab-Kombinationstherapie}

Die Resektabilität kolorektaler Lebermetastasen nach einer Behandlung mit Cetuximab in Kombination mit FOLFOX oder FOLFIRI korreliert mit einem längeren Überleben. Dabei lebten die Patienten, bei denen im Anschluss an die Cetuximab-Kombinationstherapie eine R0-Resektion durchgeführt werden konnte, signifikant länger als die Patienten, die ausschließlich die medikamentöse Kombinationstherapie erhielten. So das Ergebnis einer aktuellen Auswertung der CELIM-Studie.

In die Phase-II-Studie CELIM wurden zwischen Dezember 2004 und März 2008114 Patienten mit primär nicht resektablen kolorektalen Lebermetastasen eingeschlossen (Folprecht G et al., 2010, Lancet Oncol 11:38-47). Die Patienten erhielten randomisiert Cetuximab (Erbitux ${ }^{\circledR}$ ) plus FOLFOX (Folinsäure/5-Fluorouracil/Oxaliplatin) oder FOLFIRI (Folinsäure/5-Fluorouracil/Irinotecan). Das Tumoransprechen (RECISTKriterien, primärer Endpunkt) wurde alle acht Wochen untersucht. Zusätzlich wurde die Resektabilität von einem multidisziplinären Team nach 16 Wochen und dann in Abständen von zwei Monaten für zwei Jahre neu bewertet. Bei den auswertbaren 106 Patienten zeigten sich hohe Ansprechraten - bestätigtes partielles oder komplettes Ansprechen - von 68\% unter Cetuximab/FOLFOX und $57 \%$ unter Cetuximab/ FOLFIRI. Bei 38\% bzw. 30\% der Patienten wurde unter der CetuximabKombinationstherapie sogar eine sekundäre Resektabilität erreicht.

\section{Signifikanter Überlebensvorteil}

Die aktuelle Auswertung der Überlebensdaten in der Nachbeobachtungszeit bis Juni 2011 ergab insgesamt ein medianes Gesamtüberleben von 33,1 Monaten und eine 4-Jahres-Gesamtüberlebensrate von 28\% unter der multidisziplinären Behandlung, einschließlich der Cetuximab-Kombinationstherapie. Ein signifikanter Überlebensvorteil zeigte sich in Abhängigkeit vom R0-Resektionsstatus mit einem medianen Gesamtüberleben von 46,7 Monaten bei Patienten mit R0-Resektion und 27,3 Monaten ohne R0-Resektion ( $p=0,002 ; A b b$.).

In Abhängigkeit von der medikamentösen Therapie (Cetuximab/ FOLFOX versus Cetuximab/FOLFIRI) oder dem KRAS-Status (KRASWildtyp versus mutiertes KRAS) ergab sich kein signifikanter Unterschied bei den Überlebensdaten; numerisch lebten Patienten mit KRAS-Wildtyptumor jedoch länger als Patienten mit einer KRAS-Mu-

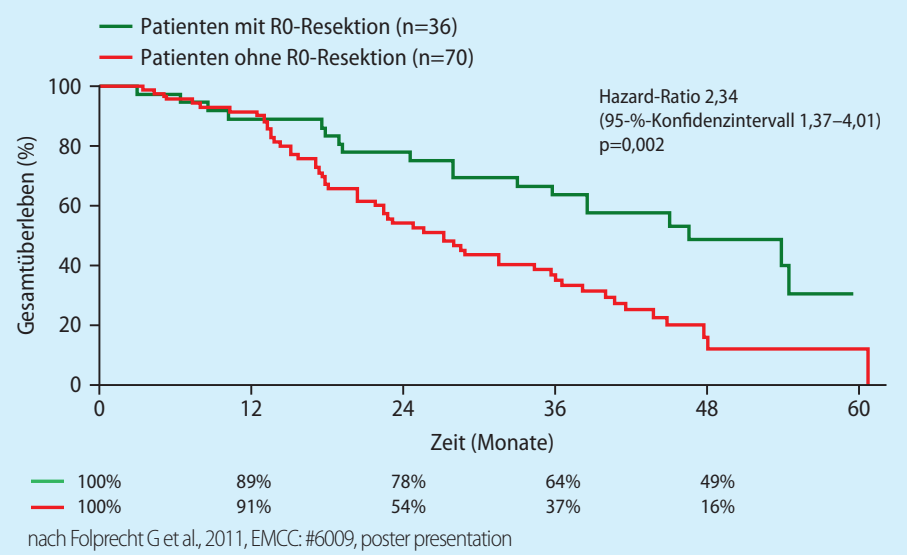

CELIM-Studie - Gesamtüberleben: R0-Resektion versus keine R0-Resektion nach Cetuximab-Kombinationstherapie

tation im Tumorgewebe (medianes Gesamtüberleben: 36,1 Monate versus 27,4 Monate; Hazard-Ratio 1,48; 95-\%-Konfidenzintervall 0,882,48).

Auf die Bedeutung der multidisziplinären Zusammenarbeit und der sorgfältigen Patientenselektion - insbesondere bezüglich der Anzahl der Metastasen - weisen laut den Autoren die Daten zum krankheitsfreien Überleben nach R0-Resektion hin: Im Median betrug das krankheitsfreie Überleben der Patienten 9,9 Monate, in Abhängigkeit von der Anzahl der Metastasen war es jedoch signifikant unterschiedlich ( $<5$ Metastasen 16,8 Monate, 5-10 Metastasen 8,2 Monate, $>10$ Metastasen 2,5 Monate; $\mathrm{p}<0,001)$. AM

Folprecht $\mathrm{G}$ et al., 2011, EMCC:\#6009, poster presentation 